\title{
Robotic Bronchoscopy for the Diagnosis of Peripheral Lung Nodules: a Review
}

\author{
Jonathan Puchalski ${ }^{1}$ \\ Accepted: 2 December 2020 / Published online: 4 January 2021 \\ (C) The Author(s) 2021
}

\begin{abstract}
Purpose of Review Conventional bronchoscopy has limitations based on an inability to accurately reach and reliably diagnose peripheral lesions with many studies having a yield of less than 50\%. Although newer technology such as virtual bronchoscopy, use of smaller bronchoscopes, peripheral endobronchial ultrasound, and electromagnetic navigation may have some improvements and a better safety profile, oftentimes transthoracic or surgical biopsies are required to establish a diagnosis and rule out malignancy. The purpose of this review is to highlight the potential benefits of robotic bronchoscopy, the latest in technological advances for this very common medical issue.

Recent Findings Recently published early studies suggest the yield of robotic bronchoscopy may surpass $90 \%$. Studies performed in cadavers and humans suggest robotic bronchoscopic platforms are better than currently existing bronchoscopic modalities for lung nodule diagnosis and have a favorable safety profile.

Summary Although additional multi-center randomized clinical trials are needed, robotic bronchoscopy appears poised to supplement current bronchoscopic techniques for establishing a diagnosis of pulmonary nodules.
\end{abstract}

Keywords Robotic bronchoscopy $\cdot$ Peripheral pulmonary nodules $\cdot$ Diagnostic bronchoscopy

\section{Introduction}

Lung cancer is the most common cause of cancer death in the world. What starts as an abnormal population of cells can, if caught in a curative stage, manifest radiographically as a pulmonary nodule. The early detection of lung nodules has shown to improve mortality and volumetric analysis may have an integral part of nodule management based on results of the NELSON study [1].

An estimated 1.6 million new pulmonary nodules will be detected by chest CT scans annually in the USA [2]. Most detected nodules are benign and it is often difficult to radiographically distinguish those that are malignant, leading to the need for biopsies. Transthoracic biopsies and surgical resection have high diagnostic yield but more complications than bronchoscopy, including pneumothorax or surgical morbidities that increase the costs of the procedures. Unfortunately,

Jonathan Puchalski

Jonathan.puchalski@yale.edu

1 Yale University School of Medicine, 15 York Street, LCI 100, New Haven, CT 06510, USA conventional bronchoscopy is notoriously poor at reliably diagnosing peripheral pulmonary nodules, particularly for those that are small, do not have an airway clearly leading to them (bronchus sign on computed tomography), are benign, and display a host of other factors. A recent prospective, randomized controlled trial comparing conventional bronchoscopy with fluoroscopic guidance to that using a thin bronchoscope and radial endobronchial ultrasound (EBUS) failed to show a statistically significant diagnostic difference between groups in patients with peripheral pulmonary lesions $1.5-5 \mathrm{~cm}$ in size [3]. Bronchoscopy was only diagnostic in $44 \%$ of patients with peripheral lesions in that study. Limitations to successful biopsy may include the ability to reach into the lung periphery and control or precision at the time of biopsy. Improved instrument stabilization at the time of biopsy may improve the diagnostic yield, particularly when an eccentric as opposed to concentric view is obtained by radial EBUS.

Various technologic advances have been developed with the intent of improving the diagnostic yield of bronchoscopy. For peripheral lesions, these include the use of navigational systems, thin and ultrathin bronchoscopy, radial endobronchial ultrasound, radiographic airway reconstruction, virtual bronchoscopy, bronchoscopic transparenchymal 
nodule access, and use of cone beam CT. While providing an improved diagnostic yield over non-guided bronchoscopic techniques, the accuracy remains close to $70 \%$, far below the 90-95\% yield of transthoracic biopsies [4]. The prospective NAVIGATE study was a multi-center cohort study that evaluated the use of the superDimension navigation system (Medtronic, Minneapolis, MN). The median lesion size was $20.0 \mathrm{~mm}$ of 1215 subjects in 29 centers. The 12-month diagnostic yield was 73\% [5]. The AQuIRE quality improvement registry reported a diagnostic yield using electromagnetic navigational (EMN) bronchoscopy of only 38.5\% [6]. A recent meta-analysis reported a sensitivity of $77 \%$ [7]. The use of cone beam CT and augmented fluoroscopy is beyond the scope of this article but entails new technologic advances regarding the diagnosis of peripheral pulmonary lesions [8]. However, the prevalence of lung nodules and current limitations of diagnostic techniques highlight the need for improvements, and robotic bronchoscopy may provide some solutions.

\section{Guided Bronchoscopic Approaches for Peripheral Nodules}

Guided bronchoscopy includes use of ultrathin bronchoscopes with peripheral EBUS, EMN bronchoscopy, and robotic bronchoscopy. The PRECISION-1 investigation [9] was a prospective, single-blinded, randomized, controlled comparative study assessing the success in localization and puncture of peripheral pulmonary nodules. This primary endpoint was verified by cone beam CT for EMN bronchoscopy and robotic bronchoscopy using the Ion ${ }^{\mathrm{TM}}$ system (Intuitive Surgical, Sunnyvale, CA) (Fig. 1). This cadaver study used implantable nodules that were distributed across all lobes, including $80 \%$ in the periphery and $50 \%$ with a bronchus sign. Preclinical study validation was performed. The mean nodule diameter was $16.5 \pm 1.5 \mathrm{~mm}$.

Proceduralists used all three guided approaches to diagnose the implanted nodules, first with ultrathin bronchoscopy and peripheral EBUS to limit virtual mapping navigational bias. A total of 60 procedures were performed to target 20 peripheral pulmonary nodules, and 20 attempts were made with each modality. During EMN bronchoscopy and robotic bronchoscopy, the proceduralists were prohibited from using peripheral EBUS. Fluoroscopy was routinely used during the procedures and cone beam CT was used at the conclusion of each attempt. The needle target distance for a maximum of three attempted passes was recorded.

Needle passes were attempted in $65 \%$ of ultrathin-radial EBUS bronchoscopy, $85 \%$ of EMN bronchoscopy, and $100 \%$ of robotic cases. The rate of successful puncture was greater in robotic bronchoscopy compared to EMN bronchoscopy $(80 \%$ vs $45 \% ; p=.02)$. There was no significant difference between ultrathin-radial EBUS bronchoscopy and EMN bronchoscopy.

Secondary analysis included whether successful localization and needle passes were in the center, peripheral, distal, or adjacent to the lesions. There was no statistically significant difference in the groups. However, when "missing" the target, measuring the distance between the nodule and the needle pass showed statistically significant longer distances using radial EBUS, EMN bronchoscopy, and robotic bronchoscopy, in favor of the latter. This study suggested that bronchoscopists were more likely to attempt biopsy and either successfully puncture or be closer to lesions when using robotic bronchoscopy compared to that with radial EBUS or electromagnetic navigation.

\section{Robotic Bronchoscopy}

The Intuitive Robotic Bronchoscope System or Ion ${ }^{\mathrm{TM}}$ (Intuitive Surgical, Sunnyvale, CA, USA) includes a planning station, system cart with monitor to display real-time visualization, and instruments including an articulating, flexible catheter. A pre-procedure CT with $1.0-\mathrm{mm}$ reconstruction slices allows virtual planning through semi-automatic pathway creation. Manual segmentation of all airways in the vicinity of the lesion can be performed if the pathway does not reach the target. The system is docked on the housing of a swivel connector connected to an endotracheal tube. At 3.5$\mathrm{mm}$ diameter, the catheter incorporates a shape-sensing fiber along its entire length, thus providing shape and positional feedback. A video probe allows live visualization while driving the catheter. Instruments that fit a 2.0-mm tool channel can be advanced after the video probe has been removed, including forceps, biopsy brushes, and a proprietary flexible biopsy needle that is extendable up to $3 \mathrm{~cm}$. The provided distance from the catheter tip to the lesion allows for controlled transbronchial needle aspiration (TBNA) needle strokelength setting and extension distance of other tools. The software provides the ultimate angle for fluoroscopy when close to the pleura, thus facilitating an accurate planar view of needle advancement. The length of the biopsy needle can be set to avoid the pleura and to reach the middle of the nodule [10].

The Robotic Endoscopy System (RES), Monarch ${ }^{\mathrm{TM}}$ (Auris Robotics, Redwood City, CA), includes the robotic endoscope, patient-side system, controller rack, and operator console (Fig. 2). The endoscope includes a video bronchoscope with an outer sheath, both of which allow 4-way steering control. This enables telescoping capability, which might enhance distal control, stability, and endoscope reach. Two robotic arms are under continuous, direct visual control to manipulate the bronchoscope and sheath using the endoscopy controller. The controller moves the robotic arms that contain rotary pulleys to drive the bronchoscope; it controls insertion, 
Fig. 1 a The Ion ${ }^{\mathrm{TM}}$ system (Intuitive Surgical) includes the system cart with monitor and the controller (b) that works similar to a computer mouse and roller. $\mathbf{c}$ The robotic catheter with a vision probe allows visualization during navigation of the airways. Images provided by Intuitive

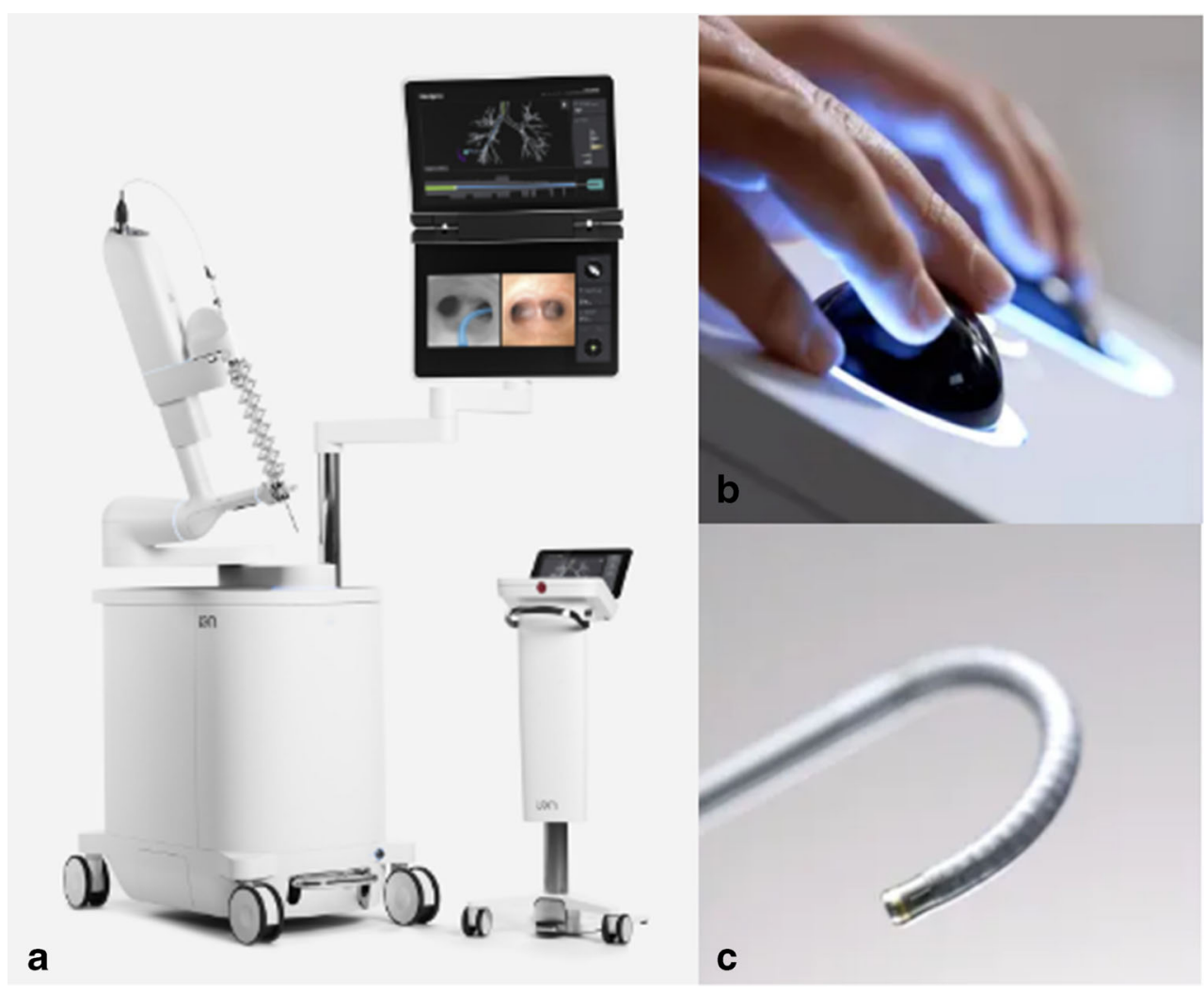

retraction, and rotation of the outer sheath and inner scope, either simultaneously or independently. The sheath is $6.0 \mathrm{~mm}$, the outer scope diameter is $4.2 \mathrm{~mm}$, and the working channel is $2.1 \mathrm{~mm}$. The distal section of the bronchoscope is advanced beyond the sheath and can deflect $180^{\circ}$ in any direction. The system uses an electromagnetic field generator and reference sensors like other electromagnetic navigational bronchoscopy

Fig. 2 The Monarch ${ }^{\mathrm{TM}}$ robotic bronchoscopy system. From: [14•] with permission from Springer and open access under terms of the creative commons license http://creativecommons. org/licenses/by/4.0. This includes an (a) outer sheath with inner scope, (b) cart with robotic arms, (c) scope attachment that allows saline irrigation and suctioning, (d) tower with monitor, and (e) the controller

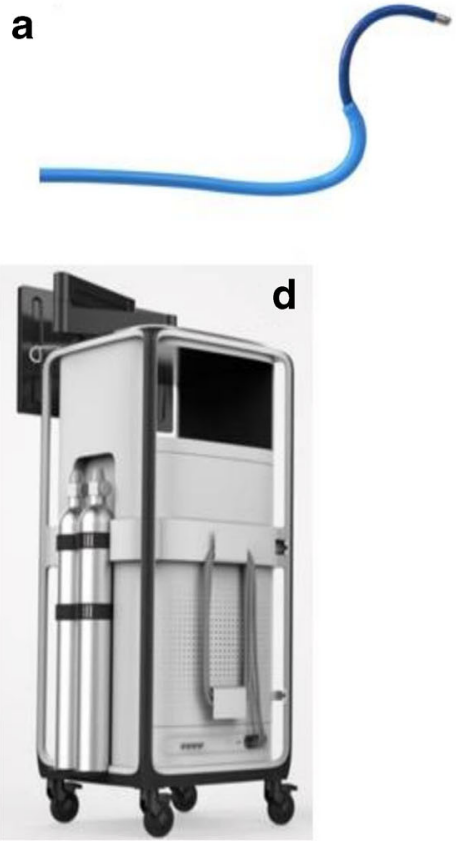

systems. A peristaltic pump and valves enable irrigation and aspiration control.

Benefits of the system may transpire from the structural support offered by the outer sheath that is locked in the target segment before advancing the scope, the ability to make subtle turns due to the 4-directional steering and due to the distal section being capable of achieving articulation in pitch and/
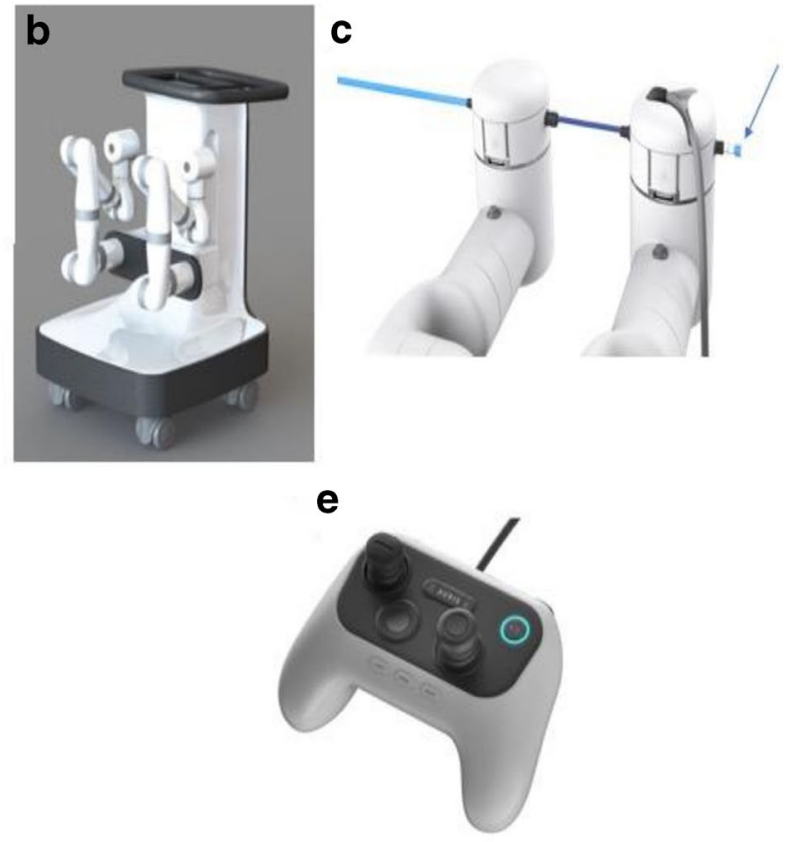
or yaw. Visualization of the peripheral airway and biopsy tools may better enable the operator to steer the tools towards the target. Torque on the bronchoscope and airway distortion are accomplished by locking the scope in position, and thus diagnostic yield may be improved compared to other tools.

\section{Cadaveric Studies of Robotic Bronchoscopy}

The PRECISION-1 study described previously [9] evaluated the spatial sensing technology of the Ion ${ }^{\mathrm{TM}}$ robotic bronchoscopy platform. Investigators performing the REACH (Robotic Endoscopic Airway Challenge) assessment compared the performance of the Robotic Endoscopy System (RES; Monarch $^{\mathrm{TM}}$, Auris Robotics) to a conventional thin bronchoscope (4.2 $\mathrm{mm}$ with $2.0-\mathrm{mm}$ working channel) to access peripheral airways within human cadaveric lungs (Fig. 3 and Table 1). Two operators used the conventional scope and the RES. The outer sheath of the RES was "parked" more proximal than the targeted bronchus given its outer diameter being larger.

Investigators advanced the conventional thin bronchoscope into the airways and advanced a guidewire to the pleura. This allowed the bronchoscope and robotic system to maneuver the same airways and to obtain distance from the pleura. Insertion depth from the main carina, airway generation count, and termination points were recorded. The robotic system advanced beyond the conventional bronchoscope in all airway generations despite similarities in size. It is hypothesized that the outer sheath design of the RES provided structural support while advancing the inner scope to the periphery. This was particularly evident in RB1 and LB1+2. In the middle and lower lobes, it was felt that the distal end of the robotic scope was able to negotiate the subtle turns at distal airway bifurcations.

The ACCESS study [2] was performed in human cadaveric lungs using the Monarch ${ }^{\mathrm{TM}}$ platform. Operators included 8 bronchoscopists, 8 cadavers, and artificial tumor targets that were implanted $0-33 \mathrm{~mm}$ from the pleura. Lesions $10-30 \mathrm{~mm}$ in diameter were created and thin-slice CT scans were performed. The bronchoscopists received prior training about system manipulation and performing biopsies. No assistance was received during the actual cases.

Direct endoscopic visualization using the scope beyond the sheath, electromagnetic navigational guidance, fluoroscopy, and peripheral EBUS were used. Biopsies were performed using a 24-G TBNA needle (Auris Health) and recorded as "diagnostic" when the pigmented mica powder in the implants was obtained. Forceps biopsies were performed at the discretion of the bronchoscopist.

The mean (16.0 mm; SD 10.6) and median $(13.8 \mathrm{~mm}$; range $0.0-35.3 \mathrm{~mm}$ ) distance from the closest edge of the target to the nearest pleural edge was measured. Fluoroscopy visualized $36 / 67(53.7 \%)$ of the nodules. The robotic scope was advanced within $27.6 \mathrm{~mm}$ (SD $8.6 \mathrm{~mm}$ ) of the targeted lesions. Radial probe visualized 59/67 (88.1\%) of nodules. Of these, $48(81.4 \%)$ were eccentric and $11(18.6 \%)$ were concentric. The diagnostic yield for all nodules was 63/67 (94\%). Transbronchial biopsies produced an additional 2 diagnoses for a final yield of $97 \%(65 / 67)$. There was no statistically significant difference in yield between nodules $\geq 20 \mathrm{~mm}$ and those smaller, nor those with an eccentric compared to concentric view. The diagnostic yield for nodules $<10 \mathrm{~mm}$ from the pleura was $18 / 20$ (90\%) compared with $47 / 47$ (100\%) of nodules $\geq 10 \mathrm{~mm}$ from the nearest pleural surface $(p=0.086)$. No obvious airway trauma was identified.

Table 1 Comparison of FDA-approved robotic bronchoscopy platforms as of Fall, 2020

\begin{tabular}{|c|c|c|}
\hline System & Ion $^{\mathrm{TM}}$ (Intuitive) & Monarch $^{\mathrm{TM}}$ (Auris) \\
\hline $\begin{array}{l}\text { Size of } \\
\text { endobronchial } \\
\text { equipment }\end{array}$ & Articulating catheter: $3.5-\mathrm{mm}$ outer diameter & $\begin{array}{l}\text { Outer sheath }(6.0 \mathrm{~mm}) \text { with inner scope }(4.2 \mathrm{~mm}) \\
\text { containing suction and a light source }\end{array}$ \\
\hline $\begin{array}{l}\text { Size of working } \\
\text { channel }\end{array}$ & $2 \mathrm{~mm}$ & $2.1 \mathrm{~mm}$ \\
\hline Endoscopic vision & Peripheral vision probe is removed prior to biopsy & $\begin{array}{l}\text { Visualizing scope remains in place during the } \\
\text { procedure }\end{array}$ \\
\hline $\begin{array}{l}\text { Navigation } \\
\text { technology }\end{array}$ & $\begin{array}{l}\text { Fiber optic "shape sensing" technology } \\
\text { Peripheral vision is removed }\end{array}$ & $\begin{array}{l}\text { Uses electromagnetic navigation and peripheral } \\
\text { vision (real-time input from micro camera) }\end{array}$ \\
\hline $\begin{array}{l}\text { Accompanying } \\
\text { technology }\end{array}$ & CT, radial EBUS, fluoroscopy, live views & CT, EMN, radial EBUS, fluoroscopy, live views \\
\hline Controller & $\begin{array}{l}\text { Trackball and scroll wheel } \\
\text { Touch screen }\end{array}$ & $\begin{array}{l}\text { Two joysticks plus various buttons for irrigation and } \\
\text { aspiration, among others } \\
\text { Touch screen monitor }\end{array}$ \\
\hline Steering and control & $\begin{array}{l}\text { Active robotic control of catheter position which corrects unwanted } \\
\text { deflection and secures a fixed position during biopsy }\end{array}$ & $\begin{array}{l}\text { 4-way steering control } \\
\text { Sheath and bronchoscope can be locked in place }\end{array}$ \\
\hline Biopsy tools & Can use needle, forceps, brushes & Can use needle, forceps, brushes \\
\hline
\end{tabular}


Fig. 3 a The articulating catheter (Ion ${ }^{\mathrm{TM}}$ ) and $\mathbf{b}$ the robotic endoscope (Monarch ${ }^{\mathrm{TM}}$ ) with (1) a camera, (2) light sources, and (3) working channel

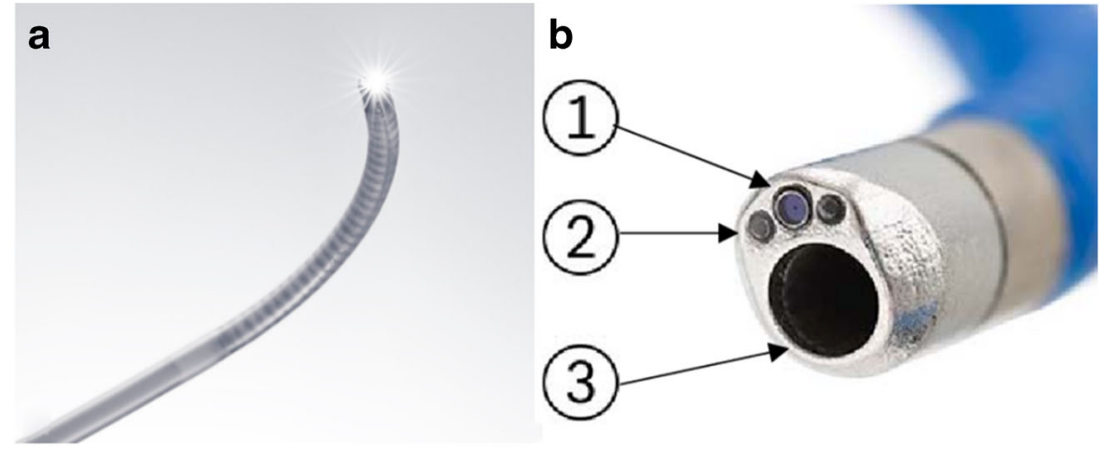

\section{Human Studies of Robotic Bronchoscopy}

Fielding et al. [10] reported the first-in-human study of safety and feasibility of the Intuitive (Ion ${ }^{\mathrm{TM}}$ ) Robotic Bronchoscope System. Two experienced operators performed the procedure in a controlled population. The primary safety endpoint included peri-procedure pneumothorax and bleeding requiring intervention. The feasibility endpoint evaluated the ability to access pulmonary nodule(s) and retrieve tissue samples from a pre-planned location based on CT imaging and identified by the planning station. Tissue samples were characterized by a histological or cytological feature other than bronchial epithelial cells or lung parenchyma.

The investigators enrolled 30 patients, of which 11 (36.7\%) had previously undergone unsuccessful biopsy attempts. In the axial dimension, $23 / 30(79.3 \%)$ nodules were $<2 \mathrm{~cm}$ in the largest diameter. The mean nodule size was $14.8 \mathrm{~mm}$ and ranges up to $26.6 \mathrm{~mm}$. Eccentric images were seen in approximately half of the radial EBUS cases and 17 (58.6\%) of the lesions had a bronchus sign visible on CT. Using radial EBUS, $93 \%$ of the nodules were visible. The catheter reached significantly more branch points than did the 4.4-mm bronchoscope $(2.21 \pm 1.2)$. Procedure time was $63.9 \pm 24.4 \mathrm{~min}$ and the time lessened with experience. The mean number of biopsy attempts was $2.6 \pm 1.8$. The primary feasibility endpoint was reached in $28(96.6 \%)$ of cases. Although not designed as a performance study, performance trends through 6month follow-up demonstrated an overall diagnostic yield of $79.3 \%(63.6-98.5 \%)$ with the sensitivity for malignancy trending towards $88.2 \%$ and specificity trending towards $63.6 \%(30.8-89.1 \%)$.

There were no instances of pneumothorax or bleeding requiring intervention observed. Notably, lesions were at least $15 \mathrm{~mm}$ from the visceral pleura. There were no complications related to the study system. One patient had a delayed re-paralysis/muscle relaxant complication and another developed contralateral pneumonia requiring hospitalization.

Rojos-Solano et al. [11] studied feasibility and complications of using the Robotic Endoscopy System (RES; Monarch $^{\mathrm{TM}}$ ). Pre-procedural CT scan parameters included 1- $\mathrm{mm}$ slice thickness at $0.5-\mathrm{mm}$ interval. Two experienced bronchoscopists performed the biopsies in 15 patients who were intubated for the procedure. The median lesion size was $2.6 \mathrm{~cm}(1.0-6.3 \mathrm{~cm})$ and median closest distance to the pleura was $0.6 \mathrm{~cm}$. An air-bronchus sign was detected on the pre-procedural CT scan. Radial EBUS was not used in this study. The bronchoscope was advanced with the endoscopy controller and navigated to the targeted segment with the aid of the CT scan and mono-planar fluoroscopy. Direct continuous visualization was used during all procedures. Biopsies were performed using a $24.5-\mathrm{G}$ needle aspiration device and SpyBite ${ }^{\mathrm{TM}}$ biopsy forceps (Boston Scientific, 4.1-mm cup opening at $55^{\circ}$ ) sequentially. Rapid on-site evaluation was routinely used and "as many needle passes and forceps biopsies" considered necessary by the cytopathologist were performed. The median number of needle aspirations was 3 ( 0 to 5) per target and the number of forceps biopsies was 7 (0 to 12). The median biopsy time decreased from $45 \mathrm{~min}$ in the first 5 cases to $20 \mathrm{~min}$. Patients had follow-up at 2 and 7 days.

There were no significant adverse events including pneumothorax or significant bleeding during the RES procedures. Three minor unrelated symptoms developed after the procedure. Tissue acquisition under direct visualization was successful in 14 of 15 (93\%) patients. The robotic tension parameters were not set appropriately in one case and conventional bronchoscopy was used to make the diagnosis and surgical biopsy was required in one non-diagnostic case. Adenocarcinoma was found in 9 patients and benign features found in 5 of the 6 other patients. The authors concluded that robotic bronchoscopy using the RES was feasible and lacked significant adverse events but acknowledged the small number of patients in the study and that the procedural team was trained prior to the procedure.

A multi-center retrospective study of 167 lesions in 165 patients was carried out by Chaddha et al. [12] using the Robotic Endoscopy System (Auris ${ }^{\mathrm{TM}}$ ). Adult patients needing guided bronchoscopy of lesions suspicious for malignancy and mycobacterial or fungal disease were enrolled in four centers. Two lesions were biopsied during the same procedure in two cases. They were excluded if conventional white light bronchoscopy identified the lesion. 
The average lesion size was $25.0 \pm 15.0 \mathrm{~mm}: 71.3 \%$ were $\leq 30 \mathrm{~mm}$ and $70.7 \%$ were located in the peripheral third of the lung. A bronchus sign was seen in $106(63.5 \%)$ of the lesions. Most $(68.5 \%)$ of the lesions were solid on imaging. Navigation was successful in $148(88.6 \%)$ of the lesions, the targeted lesions were detected with r-EBUS in $141(84.4 \%$ (eccentric $=42.5 \%$; concentric $=57.5 \%)$, and tissue samples were successfully obtained in 161 patients (97.6\%). In 3 cases, navigation was unsuccessful and in 1 case, there was software failure of the equipment. Needles (100\%) and biopsy forceps $(96 \%)$ were routinely used. The average navigation times $(17.8 \pm 19.1 \mathrm{~min})$, procedure times $(58.6 \pm 31.4 \mathrm{~min})$, and follow-up ( $185 \pm 55$ days) were recorded. Thirteen patients did not have available follow-up. The diagnostic yield ranged from 69.1 to $77 \%$ assuming patients with only inflammation on pathology had non-diagnostic and diagnostic procedures respectively.

The yield was $81.5,71.7$, and $26.9 \%$ for concentric, eccentric, and absent radial EBUS views $(p<0.001)$. Yield was higher $(78.3 \%)$ for those with compared to without $(54.1 \%)$ a bronchus sign $(p=0.001)$. Yield was not different for solid versus ground glass nodules (68.9 vs $70.6 \% ; p=0.74)$ and central versus peripheral location $(73.5 \%$ vs $67.8 \% ; p=$ $0.47)$ and did not depend on lesion size $(45.5 \%$ for $<1 \mathrm{~cm}$ vs $68.5 \%$ for $1-3 \mathrm{~cm}$ vs $77.1 \%$ for $\geq 3 \mathrm{~cm} ; p=0.11)$. Lung cancer was the most common diagnosis. "Other" benign diagnoses were chronic or granulomatous inflammation that decreased in size on follow-up imaging whereas necrotic material was felt to represent a lung abscess and histoplasmosis while atypical cells were favored to represent metastases if a patient had a concurrent malignancy.

Pneumothorax occurred in $6(3.6 \%)$ cases, requiring a chest tube in $4(2.4 \%)$. Significant bleeding requiring bronchoscopic instillation of saline was reported in $4(2.4 \%)$ cases. There were no cases requiring blood transfusion, thoracotomy, or bronchial blockers and no reports of other procedure-related complications, respiratory failure, or deaths.

The authors concluded that robotic bronchoscopy should be offered to all patients with suspicious peripheral lesions that also require concurrent guidelinesrecommended EBUS-TBNA lymph node staging for CTPET normal mediastinum or prior to stereotactic body radiotherapy (SBRT), or when preoperative tissue is required based on questionable operability, patient or surgeon's preference.

The recently published BENEFIT study was a prospective, multi-center pilot and feasibility study in humans with peripheral lesions $1-5 \mathrm{~cm}$ in size. This included 55 patients in 5 medical centers with a median lesion size of $23 \mathrm{~mm}$. Investigators successfully localized the lesion in 51/53 (96.2\%) patients using radial EBUS and the Monarch ${ }^{\mathrm{TM}}$ robotic system. TBNA and ROSE were performed with the use of transbronchial biopsies if ROSE was negative. Patients had "crossover" procedures if TBNA with ROSE was non-diagnostic, including conventional bronchoscopy with electromagnetic navigation or thin bronchoscopy with radial ultrasound. One additional patient was ultimately diagnosed via these crossover procedures. Pneumothorax occurred in two patients (one required chest tube placement). Patients were followed for at least 1 year. Investigators concluded that additional large prospective studies are needed [13].

\section{Lessons Learned}

Murgu [14•] provided insight on how to improve the beginner's performance using the Monarch ${ }^{\mathrm{TM}}$ platform. Appropriate team in-servicing and use of on-site technical support are regularly offered. During ventilation of the patient, a tidal volume of approximately $8 \mathrm{ml} / \mathrm{kg}$ ideal body weight and a PEEP of 8-10 $\mathrm{cm} \mathrm{H}_{2} 0$ are used in an effort to splint open small subsegmental airways. Recruitment maneuvers may also enable advancement of the scope beyond the 5th-6th generation without a need to inject saline. Saline may give a falsely positive radial EBUS image or disrupt cells for onsite cytology, if used. Investigators recommended not advancing the sheath beyond the 4th airway generation as it may impede maneuvering of the scope in the smaller airways. However, on occasion, they would advance biopsy forceps or the radial EBUS probe in the distal airways and use a modified Seldinger technique for advancement. Similarly, they would allow pressure equilibration between the atmosphere and target airway by opening the system to atmosphere and insufflating 30-60 $\mathrm{ml}$ of air, or relaxing the scope to allow alignment with the airway axis. This could also be accomplished by retracting and reorienting the scope.

\section{Conclusions}

Lung nodules are commonly found in medical practice and lung cancer CT screening reduces mortality. Conventional bronchoscopy is limited in its ability to diagnose peripheral nodules, and technological advances such as radial EBUS and navigational bronchoscopy have improved the yield but still have significant limitations that make them inferior to transthoracic or surgical biopsies in diagnostic accuracy. New robotic bronchoscopy platforms, including the Ion ${ }^{\mathrm{TM}}$ and Monarch $^{\mathrm{TM}}$ systems, offer promise in their ability to safely and reliably diagnose peripheral lung nodules using a bronchoscopic approach. Although in its infancy, excitement abounds regarding the possibilities of robotic bronchoscopy systems for the diagnosis and potential treatment of peripheral pulmonary lesions. 


\section{Compliance with Ethical Standards}

Conflict of Interest I, Jonathan Puchalski, declare no conflicts of interest.

Human and Animal Rights and Informed Consent This article does not contain studies with human or animal subjects. It is a review of the literature.

Open Access This article is licensed under a Creative Commons Attribution 4.0 International License, which permits use, sharing, adaptation, distribution and reproduction in any medium or format, as long as you give appropriate credit to the original author(s) and the source, provide a link to the Creative Commons licence, and indicate if changes were made. The images or other third party material in this article are included in the article's Creative Commons licence, unless indicated otherwise in a credit line to the material. If material is not included in the article's Creative Commons licence and your intended use is not permitted by statutory regulation or exceeds the permitted use, you will need to obtain permission directly from the copyright holder. To view a copy of this licence, visit http://creativecommons.org/licenses/by/4.0/.

\section{References}

Papers of particular interest, published recently, have been highlighted as:

- Of importance

1. de Koning HJ, van der Aalst CM, de Jong PA, Scholten ET, Nackaerts K, Heuvelmans MA, et al. Reduced lung-cancer mortality with volume CT screening in a randomized trial. N Engl J Med. 2020;382:503-13.

2. Chen AC, Pastis NJ, Machuzak MS, Gildea TR, Simoff MJ, Gillespie CT, et al. Accuracy of a robotic endoscopic system in cadaver models with simulated tumor targets: ACCESS study. Respiration. 2020;99:56-61.

3. Tanner NT, Yarmus L, Chen A, Wang Memoli J, Mehta HJ, Pastis NJ, et al. Standard bronchoscopy with fluoroscopy vs thin bronchoscopy and radial endobronchial ultrasound for biopsy of pulmonary lesions: a multicenter, prospective, randomized trial. Chest. 2018;154:1035-43.
4. Wang Memoli JS, Nietert PJ, Silvestri GA. Meta-analysis of guided bronchoscopy for the evaluation of the pulmonary nodule. Chest. 2012;142:385-93.

5. Folch EE, Pritchett MA, Nead MA, Bowling MR, Murgu SD, Krimsky WS, et al. Electromagnetic navigation bronchoscopy for peripheral pulmonary lesions: one-year results of the prospective, multicenter NAVIGATE study. J Thorac Oncol. 2019;14:445-58.

6. Ost DE, Ernst A, Lei X, Kovitz KL, Benzaquen S, Diaz-Mendoza J, et al. Diagnostic yield and complications of bronchoscopy for peripheral lung lesions. Results of the AQuIRE registry. Am J Respir Crit Care Med. 2016;193:68-77.

7. Folch EE, Labarca G, Ospina-Delgado D, Kheir F, Majid A, Khandhar SJ, et al. Sensitivity and safety of electromagnetic navigation bronchoscopy for lung cancer diagnosis: systematic review and meta-analysis. Chest. 2020;158:1753-69. https://doi.org/10. 1016/j.chest.2020.05.534.

8. Cheng GZ, Liu L, Nobari M, Miller R, Wahidi M. Cone beam navigation bronchoscopy: the next frontier. J Thorac Dis. 2020;12:3272-8.

9. Yarmus L, Akulian J, Wahidi M, Chen A, Steltz JP, Solomon SL, et al. A prospective randomized comparative study of three guided bronchoscopic approaches for investigating pulmonary nodules: the PRECISION-1 study. Chest. 2020;157:694-701.

10. Fielding DIK, Bashirzadeh F, Son JH, Todman M, Chin A, Tan L, et al. First human use of a new robotic-assisted fiber optic sensing navigation system for small peripheral pulmonary nodules. Respiration. 2019;98:142-50.

11. Rojas-Solano JR, Ugalde-Gamboa L, Machuzak M. Robotic bronchoscopy for diagnosis of suspected lung cancer: a feasibility study. J Bronchol Interv Pulmonol. 2018;25:168-75.

12. Chaddha U, Kovacs SP, Manley C, Hogarth DK, Cumbo-Nacheli G, Bhavani SV, et al. Robot-assisted bronchoscopy for pulmonary lesion diagnosis: results from the initial multicenter experience. BMC Pulm Med. 2019;19:243.

13. Chen AC, Pastis NJ Jr, Mahajan AK, Khandhar SJ, Simoff MJ, Machuzak MS, et al. Robotic bronchoscopy for peripheral pulmonary lesions: a multicenter pilot and feasibility study (BENEFIT). Chest. 2020. https://doi.org/10.1016/j.chest.2020.08.2047.

14. Murgu SD. Robotic assisted-bronchoscopy: technical tips and lessons learned from the initial experience with sampling peripheral lung lesions. BMC Pulm Med. 2019;19:89 This article describes tips for using the robotic bronchoscopy platform. It is a unique perspective aimed at enhancing education for "lessons learned" during initial use of the equipment.

Publisher's Note Springer Nature remains neutral with regard to jurisdictional claims in published maps and institutional affiliations. 